die Incubation kürzer ist. Jedenfalls spricht der 3. Fall dafür, dass das Fleckfieber anch noch in seinen letzten Stadien ansteckt, denn Prietzseb kam an 3. April in die Charité, als Wachsmuth (Fall 1) sich schon der Reconvalescenz näherte. Man kann aber nicht wohl ụmbin, dje Ansteckung des Prietzsch auf Wachsmuth zurïckzuführen. Denn der einzige andere Fleckfieherkranke, der sich damals auf der Abtheilung befand (Schma, Fall 2), erkrankte erst am 12. April, also nur 6 Tage früher, als Prietzsch. Am auffälligsten bleibt die Erkrankung des Leichendieners (Fall 10), der, soviel sich feststellen liess, nur mit Leichen von Fleckleberkranken in Berührung gekommen ist.

\title{
2.
}

\section{Das Alter der Syphilis in Ostasien.}

\author{
Von Rud. Virchow.
}

In der Sitzung der Berliner anthropologisehen Gesellschaft vom 15. Januar 1870 (Zeitschrift für Ethnọlogie Bd. II. S. 156) habe ich eine lieihe alter Schädel besprochen, welche Hr. Fedor Jagor aus einer Höble von Nipa-Nipa auf den Philjppinen mitgebracht hat und an welchen sich unzweidentige Spuren von Syphilis vorfinden. Diese Sache ist in doppelter Beziehung von Bedeutung, einerseits für die Bestimmung des Alters der Schädel selbst, andererseits für die Bestimmung des Alters der Syphilis in Ostasien.

Im Verfolg seiner Studien über diesen Erdtheil ist Hr. Jagor auf eine, soviel ich sehe, bis jetzt unbekannte Stelle in dem wichtigen Werke von Ant. Pigafetta (Primo viaggio intorno al globo terracqueo. Ausgabe nach dem M. S. Codex der Ambrosiana in Mailand 1800. $4^{\circ}$. p. 172) gestossen, welche beweist, dass die Krankheit schon zu Anfang des 16. Jahrhunderts die ostasiatischen Inseln erreicht hatte, also aller Wahrscheinlichkeit nach schon von den ersten Entdeckern dort eingeschleppt worden ist. Die Stelle lautet im Original:

„In tutte le ysolle bavemo trovate in questo arcipelago regnia lo mal de $\mathrm{S}$. Jop e più quivi (Timor), che in altro locho et lo chiamano for franchi, cioè mal portughese."

Anmerkung des Herausgebers:

Se il mal di San Giobbe è il mal celtico, come i più vogliono, eccol trovato velle Molucche e nelle Filippine a principio del secolo XVl, e dal vederlo chiamato mal portoghese argomentar deggiamo che da Portoghesi sea stato colà portato. .... Potrebbe per mal di $\mathrm{S}$. Giobbe interdersi la lepra non infrequente nell' Asia e nelle isole del Mar del Sud.

Die Uebersetzung lautet:

„Auf allen Inseln, die wir in diesem Archipel gefunden, berrschte die Hiobskrankheit und hier (in Timor) mehr als an anderen Orten, und man nennt sie for franchi, das ist: portugiesische Krankheit."

Archiv f. pathol. Anat. Bd. LIII. Hft. 1. 
Anmerkung des Herausgebers:

"Wenn die Hiobskrankheit die celtische Krankheit ist, wie die meisten wollen, so finden wir sie biernach in den Molukken und Filippinen zu Anfang des 16. Jahrbunderts, und da sie die portugiesische Krankheit genannt wird, müssen wir schliessen, dass sie von den Portugiesen eingeschleppt worden sei. ... Man bônnte unter Hiobskrankheit den Aussatz verstehen, der in Asien und den Inseln der Südsee nicht selten ist."

Icb bemerke, dass nach der Marginal-Notiz die Stelle bei dem Jahre 1522. angeführt ist. Schwerlich ist bei der Hiobskrankheit an Aussatz zu denken. Dieser geht in den spanisch-portugiesischen Ländern überall unter dem Namen der Lazaruskrankbeit, wäbrend der Name Hiob's sich in jener Zeit und an vielen Orten an die Syphilis knüpft.

3.

\section{Fin Fall ron Xiphopagie ans dem 10. Jahrhunderte.}

Mitgetheilt von Dr. Pertseh, Bibliothekar an der Herzogl. Bibliothek zn Gotha.

In mehreren arabischen Handschriften der Gothaer Bibliothek findet sich die Erzählung eines alten Falles von Xiphopagie, welcher mit dem Falle der siamesischen Zwillinge eine überraschende Aehnlichkeit darbietet:

1. Aus der Chronik des Dhababî ( $\dagger 748 \mathrm{~d}$ : Fl. $=1347 / 8$ nach Chr.), Gothaer Handsclir. Möller, 243, fol. $2^{b}$. Unter dem Jahre $352(=963$ n. Chr.) heisst es :

„Es übersandte ein Patriarch von Armenien dem Násireddaula ben Hamdăn zwei zusammengewachsene Männer im Alter von 25 Jahren; ihr Vater war bei ihnen. Ihre Verwachsung hatte im Magen der Seite ${ }^{1}$ ) statt; sie hatten zwei Băuche, zwei Nabel, zwei Mägen, und die. Zeiten des Hungers, des Durstes und des Urinirens waren bei ihnen verschieden. Jeder von ihnen batte zwei Schultern, zwei Arme, zwei Hände, zwei Schenkel, zwei Unterbeine und eine Harnöffnung ${ }^{2}$ ); der eine von ibnen neigte (in seinen geschlechtlichen Verhältnissen) den Frauen, der andere den bartlosen Jünglingen zu. Der Kâdbî̀ Tanțchì erzăhlt: Der eine von ibnen starb, blieb so mebrere Tage und fing an zu stinken, während sein Brader nocb lebte. Da versammelte Nàsireddaula die Aerzte, ob sie die Beiden wohl trennen könnten; aber sie konnten es nicht. Darauf wurde der Lebendige von dem Geruche des Todten krank und starb."

2. Fast wörtlich derselbe Bericht, welcher deshalb entweder von Sujûti

) sic! soll wohl heissen: an der Seite des Unterleibes. Im folgenden Berichte heisst es einfach: an der Seite.

$\left.{ }^{2}\right)$ Das gebrauchte arabische Wort, ihlìl, bedeutet: 1) das Loch des Penis oder der Brustwarze; 2) den Penis selbst. Da gleich gesagt.wird, dass der eine der weiblichen Natur zuneigte, so erscheint die erstere Bedeutung hier passender. 